\title{
Association of genetic variants with coronary artery disease and ischemic stroke in a longitudinal population-based genetic epidemiological study
}

\author{
YOSHIJI YAMADA ${ }^{1,2}$, KOTA MATSUI ${ }^{2,3}$, ICHIRO TAKEUCHI ${ }^{2,3}$ and TETSUO FUJIMAKI ${ }^{4}$
}

${ }^{1}$ Department of Human Functional Genomics, Life Science Research Center, Mie University, Tsu, Mie 514-8507; ${ }^{2}$ Core Research for Evolutional Science and Technology, Japan Science and Technology Agency, Chiyoda-ku, Tokyo 102-0075;

${ }^{3}$ Department of Scientific and Engineering Simulation, Graduate School of Engineering, Nagoya Institute of Technology, Nagoya, Aichi 466-8555; ${ }^{4}$ Department of Cardiovascular Medicine, Inabe General Hospital, Inabe, Mie 511-0428, Japan

Received February 2, 2015; Accepted February 10, 2015

DOI: 10.3892/br.2015.440

\begin{abstract}
Our previous studies identified nine genes and chromosomal region 3 q28 as susceptibility loci for myocardial infarction, ischemic stroke or chronic kidney disease by genome-wide or candidate gene association studies. As coronary artery disease (CAD) and ischemic stroke may share genetic architecture, certain genetic variants may confer susceptibility to the two diseases. The present study examined the association of 13 polymorphisms at these 10 loci with the prevalence of CAD or ischemic stroke in community-dwelling individuals, with the aim of identifying genetic variants that confer susceptibility to the two conditions. Study subjects (170 with CAD, 117 with ischemic stroke and 5,718 controls) were recruited to the Inabe Health and Longevity Study, a longitudinal genetic epidemiological study of atherosclerotic, cardiovascular and metabolic diseases. The subjects were recruited from individuals who visited for an annual health checkup and they were followed up each year (mean follow-up period, 5 years). Longitudinal analysis with a generalized estimating equation, and with adjustment for age, gender, body mass index, smoking status, the prevalence of hypertension, diabetes mellitus and dyslipidemia and the serum concentration of creatinine, revealed that $\mathrm{rs} 2074380(\mathrm{G} \rightarrow \mathrm{A})$ and $\mathrm{rs} 2074381(\mathrm{~A} \rightarrow \mathrm{G})$ of the $\alpha$-kinase 1 $(A L P K 1)$ gene and $\operatorname{rs} 8089(\mathrm{~T} \rightarrow \mathrm{G})$ of the thrombospondin 2 (THBS2) gene were significantly $\left(\mathrm{P}<2 \times 10^{-16}\right)$ associated with the prevalence of CAD, with the $A A$ genotype of rs 2074380 and $G G$ genotypes of rs2074381 and rs8089 being protective against this condition. Similar analysis revealed that rs9846911 $(\mathrm{A} \rightarrow \mathrm{G})$ at chromosome 3q28, rs2074381 of $A L P K 1$,
\end{abstract}

Correspondence to: Professor Yoshiji Yamada, Department of Human Functional Genomics, Life Science Research Center, Mie University, 1577 Kurima-machiya, Tsu, Mie 514-8507, Japan

E-mail: yamada@gene.mie-u.ac.jp

Key words: coronary artery disease, ischemic stroke, genetics, polymorphism, longitudinal study rs8089 of THBS2 and rs6046 $(\mathrm{G} \rightarrow \mathrm{A})$ of the coagulation factor VII gene were significantly $\left(\mathrm{P}<2 \times 10^{-16}\right)$ associated with the prevalence of ischemic stroke, with the $G G$ genotypes of rs9846911, rs2074381 and rs8089 and the $A A$ genotype of rs6046 being protective against this condition. ALPK1 and $T H B S 2$ may thus be susceptibility loci for CAD and ischemic stroke.

\section{Introduction}

Coronary artery disease (CAD) is an important clinical problem due to its large contribution to mortality. In the United States, the total number of individuals affected by CAD or myocardial infarction (MI) was 15.5 and 7.6 million, respectively, in 2012. The annual incidence of new or recurrent $\mathrm{MI}$ and fatal CAD was 935,000, with an annual mortality of 375,295 from these conditions, in 2011 (1). Despite recent advances in therapy, such as drug-eluting stents (2) for acute coronary syndrome, CAD remains the leading cause of fatality in the United States (1). Disease prevention is an important strategy for reducing the overall burden of CAD and the identification of biomarkers for disease risk is key for risk prediction and potential intervention to reduce the chance of future coronary events.

Stroke is a complex multifactorial disorder that is believed to result from an interaction between the genetic background of an individual and various environmental factors. It is a common and serious condition, with $\sim 795,000$ individuals experiencing a new or recurrent stroke and 128,932 fatalities from stroke-related causes in 2011 in the United States. The prevalence of stroke in the United States was 6.6 million in $2012 ; 87 \%$ were ischemic stroke, $10 \%$ were intracerebral hemorrhage and 3\% were subarachnoid hemorrhage (1). Regardless of recent advances in acute stroke therapy, stroke remains the leading cause of severe disability (3) and the fourth leading cause of fatality, after heart disease, cancer and chronic lower respiratory disease in the United States (1). The identification of biomarkers of stroke risk is important for risk prediction and intervention to avert future cerebrovascular events. 
CAD and ischemic stroke (particularly atherothrombotic cerebral infarction) are atherosclerotic diseases that share various aspects of their underlying pathogenesis, as well as several risk factors, including hypertension, diabetes mellitus, dyslipidemia, chronic kidney disease and smoking (1). Twin and family studies have shown that CAD $(4,5)$ and ischemic stroke $(6,7)$ are highly heritable, with evidence of a shared heritability for the two conditions (8). Previous genome-wide association studies (GWASs) and meta-analyses of such studies have indicated various genes and loci in the predisposition to CAD or MI (9-15) or to ischemic stroke (16-21) in Caucasian populations. Furthermore, certain genetic variants originally shown to influence the risk of CAD were also subsequently found to be associated with ischemic stroke (22-24), suggestive of a shared genetic architecture for these conditions. Although CAD and ischemic stroke may share genetic factors in Caucasian populations, the genes that confer susceptibility to the two conditions in Japanese individuals have not been identified.

Our previous studies identified nine genes and chromosomal region $3 \mathrm{q} 28$ as susceptibility loci for MI, ischemic stroke or chronic kidney disease in Japanese individuals by genome-wide (25-27) or candidate gene (28-30) association studies. The aim of the present study was to examine the association of 13 polymorphisms at these 10 loci with the prevalence of CAD or ischemic stroke, as well as to identify polymorphisms that confer susceptibility to these conditions in community-dwelling Japanese individuals.

\section{Materials and methods}

Study population. Study subjects (170 with CAD, 117 with ischemic stroke and 5,718 controls) comprised community-dwelling individuals who were recruited to a population-based cohort study in Inabe (Inabe Health and Longevity Study), Mie, Japan. The Inabe Health and Longevity Study is a longitudinal genetic epidemiological study of atherosclerotic, cardiovascular and metabolic diseases (31-37). Detailed methods for recruitment of subjects and for collection and storage of medical examination data and genomic DNA samples were described previously (31).

In the CAD analysis, 170 subjects with CAD, including 88 with MI and 5,718 controls were examined. All the subjects with CAD underwent coronary angiography. The diagnosis of CAD was based on the detection of stenosis of $>50 \%$ in any major coronary artery by coronary angiography. The diagnosis of MI was based on typical electrocardiographic changes and on increases in the serum activity of creatine kinase (MB isozyme) and in the serum concentration of troponin $\mathrm{T}$. The diagnosis was confirmed by identification of the responsible stenosis in any of the major coronary arteries or in the left main trunk by coronary angiography. For the stroke analysis, 117 subjects with ischemic stroke and 5,718 controls were examined. The diagnosis of ischemic stroke was based on the occurrence of a new and abrupt focal neurological deficit, with neurological symptoms and signs persisting for $>24 \mathrm{~h}$; it was confirmed by positive findings in computed tomography or magnetic resonance imaging (or both) of the head. Individuals with cardiogenic embolic infarction, lacunar infarction alone, transient ischemic attack, hemorrhagic stroke, cerebrovascular malformations, moyamoya disease, cerebral venous sinus thrombosis, brain tumors or traumatic cerebrovascular diseases were excluded from the study. The control individuals had no history of CAD, aortic aneurysm or peripheral arterial occlusive disease; of ischemic or hemorrhagic stroke, or other cerebrovascular diseases; or of other atherosclerotic, thrombotic, embolic or hemorrhagic disorders.

The study protocol complied with the Declaration of Helsinki and was approved by the Committees on the Ethics of Human Research of Mie University Graduate School of Medicine and Inabe General Hospital (Mie, Japan). Written informed consent was obtained from all the subjects.

Selection and genotyping of polymorphisms. The 13 single-nucleotide polymorphisms (SNPs) examined in the present study were selected from our previous genome-wide (25-27) or candidate gene (28-30) association studies as previously described (31). Wild-type (ancestral) and variant alleles of the SNPs were determined from the SNP database (dbSNP; National Center for Biotechnology Information, Bethesda, MD, USA).

Venous blood $(5 \mathrm{ml})$ was collected into tubes containing $50 \mathrm{mmol} / \mathrm{l}$ ethylenediaminetetraacetic acid (disodium salt), peripheral blood leukocytes were isolated and genomic DNA was extracted from these cells with a DNA extraction kit (SMITEST EX-R\&D; Medical and Biological Laboratories Co., Ltd, Nagoya, Japan). Genotypes of 13 SNPs were determined at G\&G Science Co., Ltd., (Fukushima, Japan) by a method that combines the polymerase chain reaction and sequence-specific oligonucleotide probes with suspension array technology (Luminex, Austin, TX, USA). Primers, probes and other conditions for genotyping of SNPs examined in the present study were previously described (31). Detailed genotyping methodology was also described previously (38).

Statistical analysis. Quantitative data were compared between subjects with CAD or ischemic stroke and controls with the unpaired Student's t-test. Categorical data were compared using the $\chi^{2}$ test. The associations of 13 SNPs to the prevalence of CAD or ischemic stroke were examined in a 5-year longitudinal cohort study. Longitudinal changes in the prevalence of CAD or ischemic stroke were compared between two groups (dominant or recessive genetic model) by a generalized estimating equation (39) with adjustment for age, gender, body mass index, smoking status, the prevalence of hypertension, diabetes mellitus and dyslipidemia, and the serum concentration of creatinine. $\mathrm{P}<0.05$ was considered to indicate a statistically significant difference. Statistical analysis was performed with R software version 3-0-2 (The R Project for Statistical Computing) and JMP Genomics version 6.0 (SAS Institute Inc., Cary, NC, USA).

\section{Results}

Subject characteristics. Characteristics of subjects with CAD or ischemic stroke and controls in the cross-sectional analysis in March 2014 are shown in Table I. Age and the frequency of males were significantly greater in subjects with CAD or ischemic stroke compared to the controls. 
Table I. Characteristics of the subjects with coronary artery disease or ischemic stroke and controls: Cross-sectional analysis in March 2014.

\begin{tabular}{|c|c|c|c|c|c|}
\hline Parameter & Controls (n) & $\begin{array}{l}\text { Coronary artery } \\
\text { disease }(n)\end{array}$ & P-value & Ischemic stroke (n) & P-value \\
\hline Number of subjects & 5718 & 170 & & 117 & \\
\hline Age, years & $53.6 \pm 12.8(5718)$ & $66.0 \pm 9.5(170)$ & $<0.0001$ & $65.2 \pm 8.1(117)$ & $<0.0001$ \\
\hline Gender, \% (male/female) & $54.7 / 45.3(5718)$ & $77.6 / 22.4(170)$ & $<0.0001$ & $68.4 / 31.6(117)$ & 0.0033 \\
\hline Height, $\mathrm{cm}$ & $162.6 \pm 9.1(5674)$ & $160.7 \pm 9.5(146)$ & 0.0155 & $159.4 \pm 9.8(109)$ & 0.0004 \\
\hline Weight, kg & $60.9 \pm 12.2(5673)$ & $61.5 \pm 11.4(145)$ & 0.6080 & $60.0 \pm 11.2(109)$ & 0.3986 \\
\hline Body mass index, $\mathrm{kg} / \mathrm{m}^{2}$ & $22.9 \pm 3.4(5673)$ & $23.7 \pm 3.1(145)$ & 0.0096 & $23.5 \pm 3.4(109)$ & 0.0893 \\
\hline Waist circumference, $\mathrm{cm}$ & $80.3 \pm 9.2(5368)$ & $84.0 \pm 9.3(119)$ & $<0.0001$ & $81.7 \pm 8.7(98)$ & 0.1471 \\
\hline Alcohol drinking, $\%$ & $48.5(5718)$ & $44.1(170)$ & 0.2565 & $43.6(117)$ & 0.2897 \\
\hline Current or former smoker, $\%$ & $45.4(5718)$ & $53.5(170)$ & 0.0348 & $49.6(117)$ & 0.3636 \\
\hline Systolic blood pressure, $\mathrm{mmHg}$ & $120 \pm 16(5670)$ & $127 \pm 17(142)$ & $<0.0001$ & $127 \pm 16(109)$ & $<0.0001$ \\
\hline Diastolic blood pressure, $\mathrm{mmHg}$ & $75 \pm 12(5670)$ & $76 \pm 11(142)$ & 0.3134 & $77 \pm 11(109)$ & 0.0478 \\
\hline Mean blood pressure, $\mathrm{mmHg}$ & $90 \pm 13(5670)$ & $93 \pm 12(142)$ & 0.0070 & $94 \pm 12(109)$ & 0.0013 \\
\hline Ocular tension, right, $\mathrm{mmHg}$ & $13.5 \pm 3.0(1987)$ & $13.7 \pm 2.9(26)$ & 0.7251 & $13.0 \pm 2.9(36)$ & 0.2924 \\
\hline Functional vital capacity, 1 & $3.31 \pm 0.81(2164)$ & $2.96 \pm 0.84(28)$ & 0.0228 & $3.04 \pm 0.83(41)$ & 0.0346 \\
\hline FEV1\% & $81.4 \pm 6.5(2164)$ & $77.4 \pm 9.7(28)$ & 0.0014 & $79.5 \pm 6.2(41)$ & 0.0729 \\
\hline Serum albumin, $\mathrm{g} / \mathrm{l}$ & $44.7 \pm 2.6(3956)$ & $43.5 \pm 3.9(134)$ & $<0.0001$ & $43.7 \pm 3.3(103)$ & $<0.0001$ \\
\hline Serum total cholesterol, $\mathrm{mmol} / \mathrm{l}$ & $5.23 \pm 0.88(5656)$ & $4.68 \pm 1.00(162)$ & $<0.0001$ & $5.15 \pm 0.90(111)$ & 0.2983 \\
\hline Serum triglycerides, $\mathrm{mmol} / \mathrm{l}$ & $1.25 \pm 0.86(5647)$ & $1.43 \pm 0.94(159)$ & 0.0089 & $1.41 \pm 1.05(108)$ & 0.0656 \\
\hline Serum HDL-cholesterol, mmol/1 & $1.66 \pm 0.45(5646)$ & $1.48 \pm 0.46(159)$ & $<0.0001$ & $1.59 \pm 0.46(107)$ & 0.0894 \\
\hline Serum LDL-cholesterol, mmol/1 & $3.18 \pm 0.80(5644)$ & $2.74 \pm 0.81(159)$ & $<0.0001$ & $3.17 \pm 0.73(107)$ & 0.8378 \\
\hline Fasting plasma glucose, $\mathrm{mmol} / \mathrm{l}$ & $5.55 \pm 1.10(5659)$ & $6.45 \pm 1.85(160)$ & $<0.0001$ & $6.17 \pm 1.92(115)$ & $<0.0001$ \\
\hline Blood hemoglobin A1c, \% & $5.67 \pm 0.65(4215)$ & $6.10 \pm 1.24(129)$ & $<0.0001$ & $5.96 \pm 1.23(100)$ & $<0.0001$ \\
\hline Blood urea nitrogen, $\mathrm{mmol} / \mathrm{l}$ & $5.13 \pm 1.82(3845)$ & $6.57 \pm 3.83(139)$ & $<0.0001$ & $6.72 \pm 3.86(101)$ & $<0.0001$ \\
\hline Serum creatinine, $\mu \mathrm{mol} / 1$ & $71.2 \pm 66.6(5278)$ & $115.0 \pm 179.3(163)$ & $<0.0001$ & $147.1 \pm 267.5(114)$ & $<0.0001$ \\
\hline 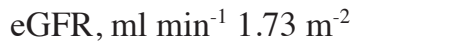 & $77.3 \pm 16.0(5278)$ & $65.6 \pm 24.8(163)$ & $<0.0001$ & $64.7 \pm 25.0(114)$ & $<0.0001$ \\
\hline Serum uric acid, $\mu \mathrm{mol} / 1$ & $325 \pm 85(5238)$ & $357 \pm 101(161)$ & $<0.0001$ & $350 \pm 87(113)$ & 0.0017 \\
\hline Serum C-reactive protein, $\mu \mathrm{g} / \mathrm{l}$ & $1.125 \pm 6.435(2006)$ & $2.063 \pm 4.809(48)$ & 0.3162 & $2.042 \pm 5.809(48)$ & 0.3285 \\
\hline White blood cells, $10^{3}$ cells $/ \mu 1$ & $5.36 \pm 1.67(4415)$ & $6.19 \pm 1.79(106)$ & $<0.0001$ & $5.34 \pm 1.54(73)$ & 0.8986 \\
\hline Red blood cells, $10^{4}$ cells $/ \mu 1$ & $437 \pm 44(4430)$ & $423 \pm 52(107)$ & 0.0006 & $419 \pm 44(73)$ & 0.0005 \\
\hline Hemoglobin, g/l & $138 \pm 15(4430)$ & $134 \pm 17(107)$ & 0.0097 & $133 \pm 17(73)$ & 0.0035 \\
\hline Hematocrit, \% & $40.3 \pm 4.2(4425)$ & $39.3 \pm 4.7(107)$ & 0.0221 & $38.8 \pm 4.6(73)$ & 0.0027 \\
\hline Platelets, $10^{4}$ cells $/ \mu 1$ & $22.4 \pm 5.4(4378)$ & $20.8 \pm 5.9(104)$ & 0.0040 & $20.6 \pm 4.4(73)$ & 0.0053 \\
\hline
\end{tabular}

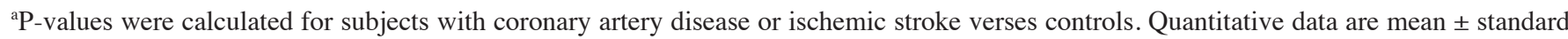
deviation. FEV1\%, forced expiratory volume in $1 \mathrm{sec}$; HDL, high-density lipoprotein; LDL, low-density lipoprotein; eGFR, estimated glomerular filtration rate $\left(\mathrm{ml} \mathrm{min}^{-1} 1.73 \mathrm{~m}^{-2}\right)=194 \times$ [age (years) $]^{-0.287} \mathrm{x}$ [serum creatinine $\left.(\mathrm{mg} / \mathrm{dl})\right]^{-1.094}[\mathrm{x} 0.739$ if female].

Associations with CAD or ischemic stroke. The association of the 13 SNPs with the prevalence of CAD or ischemic stroke was analyzed with a generalized estimating equation with adjustment for age, gender, body mass index, smoking status, the prevalence of hypertension, diabetes mellitus and dyslipidemia, and the serum concentration of creatinine. In the CAD analysis, rs2074380 $(\mathrm{G} \rightarrow \mathrm{A})$ and rs2074381 $(\mathrm{A} \rightarrow \mathrm{G})$ of the $\alpha$-kinase 1 gene $(A L P K 1)$, as well as $\operatorname{rs} 8089(\mathrm{~T} \rightarrow \mathrm{G})$ of the thrombospondin 2 gene (THBS2) were significantly $(\mathrm{P}<0.05$, recessive model) associated with the prevalence of CAD (Table II). Genotype distributions for rs2074380, rs2074381 and rs8089 in subjects with CAD and controls in a 5-year longitudinal study are shown in Table III. The $A A$ genotype of rs2074380 and the $G G$ genotypes of rs2074381 and rs8089 were protective against CAD. In the stroke analysis, rs2116519 $(\mathrm{C} \rightarrow \mathrm{T})$ of the family with sequence similarity 78-member $\mathrm{B}$ gene $(F A M 78 B)$, rs9846911 $(\mathrm{A} \rightarrow \mathrm{G})$ at chromosome $3 \mathrm{q} 28$, rs2074381 $(\mathrm{A} \rightarrow \mathrm{G})$ of $A L P K 1, \mathrm{rs} 8089(\mathrm{~T} \rightarrow \mathrm{G})$ of $T H B S 2$ and rs6046 $(\mathrm{G} \rightarrow \mathrm{A})$ of the coagulation factor VII $(F 7)$ gene were significantly $(\mathrm{P}<0.05$, recessive model $)$ associated with the prevalence of ischemic stroke (Table IV). Genotype distributions for these SNPs in subjects with ischemic stroke and controls in a 5-year longitudinal study are shown in Table V. The $C C$ genotype of rs $2116519, G G$ genotypes of rs9846911, rs2074381 and rs8089 and the $A A$ genotype of rs6046 were all protective against ischemic stroke. 
Table II. Association of the polymorphisms with coronary artery disease analyzed for 5-year longitudinal data with a generalized estimating equation.

\begin{tabular}{llcc}
\hline Gene or locus & \multicolumn{1}{c}{ SNP } & P-value & P-value \\
\hline FAM78B & rs2116519 $(\mathrm{C} \rightarrow \mathrm{T})$ & 0.2772 & 0.4233 \\
$3 q 28$ & $\mathrm{rs} 9846911(\mathrm{~A} \rightarrow \mathrm{G})$ & 0.6347 & 0.8463 \\
ALPK1 & $\mathrm{rs} 2074379(\mathrm{G} \rightarrow \mathrm{A})$ & 0.6941 & 0.7745 \\
ALPK1 & $\mathrm{rs} 2074380(\mathrm{G} \rightarrow \mathrm{A})$ & 0.9127 & $<2.0 \times 10^{-16 c}$ \\
ALPK1 & $\mathrm{rs} 2074381(\mathrm{~A} \rightarrow \mathrm{G})$ & 0.8270 & $<2.0 \times 10^{-16 c}$ \\
ALPK1 & $\mathrm{rs} 2074388(\mathrm{G} \rightarrow \mathrm{A})$ & 0.6039 & 0.6407 \\
BTN2A1 & $\mathrm{rs} 6929846(\mathrm{~T} \rightarrow \mathrm{C})$ & 0.2462 & 0.7324 \\
THBS2 & $\mathrm{rs} 8089(\mathrm{~T} \rightarrow \mathrm{G})$ & 0.2628 & $<2.0 \times 10^{-16 c}$ \\
PDX1 & $\mathrm{rs} 146021107(\mathrm{G} \rightarrow-)$ & 0.5733 & 0.9329 \\
F7 & $\mathrm{rs} 6046(\mathrm{G} \rightarrow \mathrm{A})$ & 0.3250 & 0.3275 \\
LLGL2 & $\mathrm{rs} 1671021(\mathrm{G} \rightarrow \mathrm{A})$ & 0.5494 & 0.3309 \\
ILF3 & $\mathrm{rs} 2569512(\mathrm{G} \rightarrow \mathrm{A})$ & 0.5091 & 0.3569 \\
CELSR1 & $\mathrm{rs6007897}(\mathrm{C} \rightarrow \mathrm{T})$ & 0.6664 & $\mathrm{ND}$ \\
\hline
\end{tabular}

Prevalence of coronary artery disease was compared between the two groups (dominant or recessive model) for each polymorphism with adjustment for age, gender, body mass index, smoking status, prevalence of hypertension, diabetes mellitus and dyslipidemia, and the serum concentration of creatinine. ${ }^{a}$ Dominant model: $A A$ vs. $A B+B B$ ( $A$, major allele; $B$, minor allele); ${ }^{b}$ recessive model $(A A+A B$ vs. $B B)$; ${ }^{\mathrm{b}} \mathrm{P}<0.05$. SNP, single-nucleotide polymorphism; ND, not determined.

Table III. Genotype distributions for rs2074380 and rs2074381 of $A L P K 1$ and rs8089 of THBS2 among subjects with coronary artery disease and controls.

\section{Coronary artery}

Gene (SNP) Genotype disease, n (\%) Controls, n (\%)

\begin{tabular}{lccc}
\hline$A L P K 1$ & $G G$ & $359(88.6)$ & $20,190(84.8)$ \\
$(\mathrm{rs} 2074380, \mathrm{G} \rightarrow \mathrm{A})$ & $G A$ & $46(11.4)$ & $3,469(14.6)$ \\
& $A A$ & $0(0.0)$ & $162(0.7)$ \\
ALPK1 & $A A$ & $359(88.6)$ & $20,375(85.5)$ \\
$(\mathrm{rs} 2074381, \mathrm{~A} \rightarrow \mathrm{G})$ & $A G$ & $46(11.4)$ & $3,304(13.9)$ \\
& $G G$ & $0(0.0)$ & $142(0.6)$ \\
THBS2 & $T T$ & $354(87.4)$ & $19,744(82.9)$ \\
$(\mathrm{rs} 8089, \mathrm{~T} \rightarrow \mathrm{G})$ & $T G$ & $51(12.6)$ & $3,865(16.2)$ \\
& $G G$ & $0(0.0)$ & $212(0.9)$ \\
\hline
\end{tabular}

SNP, single-nucleotide polymorphism.

\section{Discussion}

Atherosclerosis is the main cause of CAD and ischemic stroke. The principal and treatable risk factors include hypertension, diabetes mellitus, dyslipidemia, chronic kidney disease and smoking (1). In addition to these conventional risk factors, genetic variants are important in the pathogenesis of CAD $(4,5)$ and ischemic stroke $(6,7)$. Prediction of the risk for these conditions beyond the usual clinical risk factors is required
Table IV. Association of the polymorphisms with ischemic stroke analyzed for 5-year longitudinal data with a generalized estimating equation.

\begin{tabular}{|c|c|c|c|}
\hline Gene or locus & SNP & P-value & P-value ${ }^{b}$ \\
\hline$F A M 78 B$ & rs2116519 $(\mathrm{C} \rightarrow \mathrm{T})$ & 0.7794 & $0.0251^{\mathrm{c}}$ \\
\hline $3 q 28$ & rs9846911 $(\mathrm{A} \rightarrow \mathrm{G})$ & 0.9210 & $<2.0 \times 10^{-16 c}$ \\
\hline$A L P K 1$ & $\operatorname{rs} 2074379(\mathrm{G} \rightarrow \mathrm{A})$ & 0.5019 & 0.3323 \\
\hline$A L P K 1$ & $\operatorname{rs} 2074380(\mathrm{G} \rightarrow \mathrm{A})$ & 0.9588 & 0.4029 \\
\hline$A L P K 1$ & rs2074381 $(A \rightarrow G)$ & 0.9747 & $<2.0 \times 10^{-16 c}$ \\
\hline ALPK1 & $\operatorname{rs} 2074388(\mathrm{G} \rightarrow \mathrm{A})$ & 0.5072 & 0.3244 \\
\hline$B T N 2 A 1$ & rs6929846 $(\mathrm{T} \rightarrow \mathrm{C})$ & 0.9342 & 0.6411 \\
\hline$T H B S 2$ & rs8089 $(\mathrm{T} \rightarrow \mathrm{G})$ & 0.8363 & $<2.0 \times 10^{-16 c}$ \\
\hline$P D X 1$ & rs $146021107(\mathrm{G} \rightarrow-)$ & 0.4115 & 0.5500 \\
\hline$F 7$ & $\operatorname{rs} 6046(\mathrm{G} \rightarrow \mathrm{A})$ & 0.4909 & $<2.0 \times 10^{-16 c}$ \\
\hline$L L G L 2$ & $\operatorname{rs} 1671021(\mathrm{G} \rightarrow \mathrm{A})$ & 0.5353 & 0.4415 \\
\hline$I L F 3$ & $\operatorname{rs} 2569512(\mathrm{G} \rightarrow \mathrm{A})$ & 0.4175 & 0.5384 \\
\hline CELSR1 & $\operatorname{rs} 6007897(\mathrm{C} \rightarrow \mathrm{T})$ & 0.2863 & ND \\
\hline
\end{tabular}

Prevalence of ischemic stroke was compared between the two groups (dominant or recessive model) for each polymorphism with adjustment for age, gender, body mass index, smoking status, prevalence of hypertension, diabetes mellitus and dyslipidemia, and the serum concentration of creatinine. ${ }^{\mathrm{a} D o m i n a n t}$ model: $A A$ vs. $A B+B B$ ( $A$, major allele; $B$, minor allele); ${ }^{b}$ recessive model $(A A+A B$ vs. $B B)$; ${ }^{\circ} \mathrm{P}<0.05$. SNP, single-nucleotide polymorphism; ND, not determined.

Table V. Genotype distributions for five polymorphisms among subjects with ischemic stroke and controls.

\begin{tabular}{|c|c|c|c|}
\hline $\begin{array}{l}\text { Gene or locus } \\
\text { (SNP) }\end{array}$ & Genotype & $\begin{array}{c}\text { Ischemic } \\
\text { stroke, n (\%) }\end{array}$ & Controls, n (o \\
\hline \multirow{3}{*}{$\begin{array}{l}F A M 78 B \\
(\mathrm{rs} 2116519, \mathrm{C} \rightarrow \mathrm{T})\end{array}$} & $T T$ & $110(32.4)$ & $7,293(30.5)$ \\
\hline & $T C$ & $198(58.4)$ & $11,991(50.2)$ \\
\hline & $C C$ & $31(9.1)$ & $4,603(19.3)$ \\
\hline \multirow{3}{*}{$\begin{array}{l}3 q 28 \\
(\mathrm{rs} 9846911, \mathrm{~A} \rightarrow \mathrm{G})\end{array}$} & $A A$ & 293 (86.4) & $20,718(86.7)$ \\
\hline & $A G$ & $46(13.6)$ & $3,022(12.7)$ \\
\hline & $G G$ & $0(0.0)$ & $147(0.6)$ \\
\hline \multirow{3}{*}{$\begin{array}{l}A L P K 1 \\
(\mathrm{rs} 2074381, \mathrm{~A} \rightarrow \mathrm{G})\end{array}$} & $A A$ & $282(83.2)$ & $20,452(85.6)$ \\
\hline & $A G$ & $57(16.8)$ & 3,293 (13.8) \\
\hline & $G G$ & $0(0.0)$ & $142(0.6)$ \\
\hline \multirow{3}{*}{$\begin{array}{l}\text { THBS2 } \\
(\mathrm{rs} 8089, \mathrm{~T} \rightarrow \mathrm{G})\end{array}$} & $T T$ & 296 (87.3) & $19,802(82.9)$ \\
\hline & $T G$ & $43(12.7)$ & $3,873(16.2)$ \\
\hline & $G G$ & $0(0.0)$ & $212(0.9)$ \\
\hline \multirow{3}{*}{$\begin{array}{l}F 7 \\
(\text { rs6046, G } \rightarrow \text { A })\end{array}$} & $G G$ & $282(83.2)$ & $20,829(87.2)$ \\
\hline & $G A$ & $57(16.8)$ & $2,953(12.4)$ \\
\hline & $A A$ & $0(0.0)$ & $105(0.4)$ \\
\hline
\end{tabular}

SNP, single-nucleotide polymorphism.

as genetic variants would be useful for deciding how aggressively to target the risk factors that are currently responsive to treatment. The present study showed that rs2074381 $(\mathrm{A} \rightarrow \mathrm{G})$ 
of ALPK1 and rs8089 $(\mathrm{T} \rightarrow \mathrm{G})$ of THBS2 were significantly associated with the prevalence of CAD and ischemic stroke in community-dwelling Japanese individuals.

ALPK1 functions in apical transport by phosphorylating myosin 1a in epithelial cells and is indicated in the regulation of intracellular trafficking processes by phosphorylation (40). ALPK1 may act synergistically with monosodium urate monohydrate crystals to promote the production of proinflammatory cytokines through the activation of nuclear factor- $\mathrm{\kappa B}$ and mitogen-activated protein kinase (extracellular signal-regulated kinase $1 / 2$ and p38) signaling in cultured human embryonic kidney 293 (HEK293) cells, suggesting that ALPK1 may contribute to the inflammatory process associated with the development of gout (41). Our previous GWAS for chronic kidney disease showed that the overexpression of ALPK1 resulted in upregulation of the expression of cystatin C in cultured HEK293T cells (27). Cystatin C is an inhibitor of cysteine proteases and is recognized as a sensitive marker of renal dysfunction (42). Cystatin $C$ is also associated with inflammation regardless of renal function. The serum concentration of cystatin $\mathrm{C}$ was thus associated with those of C-reactive protein and fibrinogen in 990 subjects with coronary heart disease in the Heart and Soul Study (43), as well as in subjects with renal dysfunction in the Cardiovascular Health Study (44). Furthermore, the serum concentration of cystatin $\mathrm{C}$ was associated with the prevalence and severity of CAD (45-47), the risk of secondary cardiovascular events $(48,49)$ and cardiovascular mortality $(50)$. Cystatin C was also associated with ischemic and hemorrhagic stroke (51) and subclinical cerebral infarction (52). These observations suggest that the association of $A L P K 1$ with CAD and ischemic stroke may be attributable, at least in part, to the effects of cystatin $\mathrm{C}$ on the development of atherosclerosis. rs 2074381 of $A L P K 1$ was significantly associated with CAD and ischemic stroke, with the $G G$ genotype being protective against these conditions. These previous observations $(27,41,43-52)$ and our present results suggest that $A L P K 1$ may contribute to the development of CAD and ischemic stroke through the acceleration of vascular inflammation, although the molecular mechanisms underlying the role of rs2074381 of ALPK1 in the pathogenesis of CAD and ischemic stroke remain unknown.

Thrombospondins are calcium-binding glycoproteins of the extracellular matrix. They support cell attachment through interactions with multiple cell-adhesion receptors, bind to other extracellular matrix components and regulate cell shape, adhesion and migration, which in turn influence more complex biological processes, such as angiogenesis and wound healing (53). THBS2 is a multifunctional protein that contributes to the control of smooth muscle cell growth in an autocrine manner (54). THBS2 has a role in extracellular matrix organization, as suggested by the observation that disruption of THBS2 in mice results in a complex phenotype characterized by fibroblast, connective tissue and blood vessel abnormalities that are similar to those associated with Ehlers-Danlos syndrome type IV (55). Furthermore, a two-fold increase in matrix metalloproteinase 2 (MMP2) activity was found to contribute to the adhesive defect that manifested in THBS2-null fibroblasts (56). Loss of THBS2 may result in an increase in MMP2 levels in the pericellular environment, as THBS2 is capable of binding the pro- and mature forms of this proteinase (57). A $\mathrm{T} \rightarrow \mathrm{G}$ variant in the 3 ' untranslated region of THBS2 was previously shown to have a protective effect against familial premature MI in the United States, with individuals homozygous for the variant having an odds ratio of 0.31 (58). The $G$ allele of this polymorphism was also associated with a reduced risk of premature MI among individuals in the Netherlands, possibly as a result of an increase in MMP2 activity (59). In the present study, rs8089 of THBS2 was significantly associated with CAD and ischemic stroke, with the $G G$ genotype being protective against these conditions. Previous studies (53-59) and our present observations suggest that the interaction between THBS2 and MMP2 may play an important role in the predisposition to CAD and ischemic stroke, although the molecular mechanisms underlying the role of rs8089 of THBS2 in the pathogenesis of these conditions remain unclear.

rs 2116519 of $F A M 78 B$, rs 9846911 at $3 \mathrm{q} 28$ and rs6046 of $F 7$ were also associated with the prevalence of ischemic stroke. $F A M 78 B$ is located at $1 \mathrm{q} 24.1$, which was previously suggested to harbor susceptibility loci for hypertension (60) and type 2 diabetes mellitus (61), which are important risk factors for ischemic stroke, although the function of the gene remains unclear. The rs9846911 SNP at 3q28 was previously identified as a susceptibility locus for chronic kidney disease, which is also a risk factor for ischemic stroke in Japanese individuals (27). The functional role of this chromosomal region remains to be elucidated. F7 encodes coagulation factor VII, which is a vitamin K-dependent factor essential for hemostasis. The $\operatorname{rs} 6046(\mathrm{G} \rightarrow \mathrm{A}$, Arg353Gln) SNP of $F 7$ was previously associated with the levels of factor VII antigen and activity (62), as well as with prothrombin time (63). Our previous study showed that rs6046 of $F 7$ was associated with $\mathrm{MI}$ in individuals with chronic kidney disease, with the minor $A$ allele being protective against this condition, in a hospital-based study (29). Our present results are thus consistent with this previous observation (29).

There are limitations to the present study: i) As the study subjects comprised only Japanese individuals, further studies will be required in other ethnic groups. ii) rs2074381 of ALPK1 or rs8089 of THBS2 are possibly in linkage disequilibrium with other polymorphisms in the same gene or in nearby genes that are actually responsible for the development of CAD or ischemic stroke. iii) The functional relevance of rs2074381 or rs8089 to the pathogenesis of CAD or ischemic stroke remains unclear.

In conclusion, the present results suggest that $A L P K 1$ and THBS2 may be susceptibility loci for CAD and ischemic stroke in Japanese individuals. Determination of the genotype for rs2074381 and rs8089 may prove informative for assessment of the genetic risk for CAD and ischemic stroke in such individuals.

\section{Acknowledgements}

The present study was supported by the Core Research for Evolutional Science and Technology of the Japan Science and Technology Agency (to Y.Y. and I.T.), as well as by a Grant-in-Aid for Scientific Research from the Ministry of Education, Culture, Sports, Science and Technology of Japan (grant no. 24590746 to Y.Y.). 


\section{References}

1. Mozaffarian D, Benjamin EJ, Go AS, et al: Heart disease and stroke statistics-2015 update: A report from the american heart association. Circulation 131: e29-e322, 2015.

2. Stefanini GG and Holmes DR Jr: Drug-eluting coronary-artery stents. N Engl J Med 368: 254-265, 2013.

3. Donnan GA, Fisher M, Macleod M and Davis SM: Stroke. Lancet 371: 1612-1623, 2008.

4. Arnett DK, Baird AE, Barkley RA, et al; American Heart Association Council on Epidemiology and Prevention; American Heart Association Stroke Council; Functional Genomics and Translational Biology Interdisciplinary Working Group: Relevance of genetics and genomics for prevention and treatment of cardiovascular disease: A scientific statement from the American Heart Association Council on Epidemiology and Prevention, the Stroke Council and the Functional Genomics and Translational Biology Interdisciplinary Working Group. Circulation 115: 2878-2901, 2007.

5. Roberts R and Stewart AF: The genetics of coronary artery disease. Curr Opin Cardiol 27: 221-227, 2012.

6. Hassan A and Markus HS: Genetics and ischaemic stroke. Brain 123: 1784-1812, 2000.

7. Dichgans M: Genetics of ischaemic stroke. Lancet Neurol 6 : 149-161, 2007.

8. Banerjee A, Lim CC, Silver LE, Welch SJ, Banning AP and Rothwell PM: Familial history of stroke is associated with acute coronary syndromes in women. Circ Cardiovasc Genet 4: 9-15, 2011.

9. Wellcome Trust Case Control Consortium: Genome-wide association study of 14,000 cases of seven common diseases and 3,000 shared controls. Nature 447: 661-678, 2007.

10. Helgadottir A, Thorleifsson G, Manolescu A, et al: A common variant on chromosome 9p21 affects the risk of myocardial infarction. Science 316: 1491-1493, 2007.

11. McPherson R, Pertsemlidis A, Kavaslar N, et al: A common allele on chromosome 9 associated with coronary heart disease. Science 316: 1488-1491, 2007.

12. Samani NJ, Erdmann J, Hall AS, et al; WTCCC and the Cardiogenics Consortium: Genomewide association analysis of coronary artery disease. N Engl J Med 357: 443-453, 2007.

13. Peden JF, Hopewell JC, Saleheen D, et al; Coronary Artery Disease (C4D) Genetics Consortium: A genome-wide association study in Europeans and South Asians identifies five new loci for coronary artery disease. Nat Genet 43: 339-344, 2011.

14. Schunkert H, König IR, Kathiresan S, et al; Cardiogenics; CARDIoGRAM Consortium: Large-scale association analysis identifies 13 new susceptibility loci for coronary artery disease. Nat Genet 43: 333-338, 2011.

15. Deloukas P,KanoniS, Willenborg C, etal; CARDIoGRAMplusC4D Consortium; DIAGRAM Consortium; CARDIOGENICS Consortium; MuTHER Consortium; Wellcome Trust Case Control Consortium: Large-scale association analysis identifies new risk loci for coronary artery disease. Nat Genet 45: 25-33, 2013

16. Gretarsdottir S, Thorleifsson G, Manolescu A, et al: Risk variants for atrial fibrillation on chromosome $4 \mathrm{q} 25$ associate with ischemic stroke. Ann Neurol 64: 402-409, 2008.

17. Ikram MA, Seshadri S, Bis JC, et al: Genomewide association studies of stroke. N Engl J Med 360: 1718-1728, 2009.

18. Gudbjartsson DF, Holm H, Gretarsdottir S, et al: A sequence variant in ZFHX3 on 16q22 associates with atrial fibrillation and ischemic stroke. Nat Genet 41: 876-878, 2009.

19. Bellenguez C, Bevan S, Gschwendtner A, et al; International Stroke Genetics Consortium (ISGC); Wellcome Trust Case Control Consortium 2 (WTCCC2): Genome-wide association study identifies a variant in HDAC9 associated with large vesse ischemic stroke. Nat Genet 44: 328-333, 2012.

20. Traylor M,Farrall M,Holliday EG, et al; Australian Stroke Genetics Collaborative, Wellcome Trust Case Control Consortium 2 (WTCCC2); International Stroke Genetics Consortium: Genetic risk factors for ischaemic stroke and its subtypes (the METASTROKE collaboration): A meta-analysis of genome-wide association studies. Lancet Neurol 11: 951-962, 2012.

21. Kilarski LL, Achterberg S, Devan WJ, et al; GARNET Collaborative Research Group, Wellcome Trust Case Control Consortium 2, Australian Stroke Genetic Collaborative, the METASTROKE Consortium, and the International Stroke Genetics Consortium: Meta-analysis in more than 17,900 cases of ischemic stroke reveals a novel association at $12 \mathrm{q} 24.12$. Neurology 83: 678-685, 2014.
22. Gschwendtner A, Bevan S, Cole JW, et al; International Stroke Genetics Consortium: Sequence variants on chromosome 9p21.3 confer risk for atherosclerotic stroke. Ann Neurol 65: 531-539, 2009.

23. Williams FM, Carter AM, Hysi PG, et al; EuroCLOT Investigators; Wellcome Trust Case Control Consortium 2; MOnica Risk, Genetics, Archiving and Monograph; MetaStroke; International Stroke Genetics Consortium: Ischemic stroke is associated with the ABO locus: The EuroCLOT study. Ann Neurol 73: 16-31, 2013.

24. Dichgans M, Malik R, König IR, et al; METASTROKE Consortium; CARDIoGRAM Consortium; C4D Consortium; International Stroke Genetics Consortium: Shared genetic susceptibility to ischemic stroke and coronary artery disease: A genome-wide analysis of common variants. Stroke 45: 24-36, 2014.

25. Yamada Y, Nishida T, Ichihara S, et al: Association of a polymorphism of BTN2A1 with myocardial infarction in East Asian populations. Atherosclerosis 215: 145-152, 2011.

26. Yamada Y, Fuku N, Tanaka M, et al: Identification of CELSR1 as a susceptibility gene for ischemic stroke in Japanese individuals by a genome-wide association study. Atherosclerosis 207: 144-149, 2009.

27. Yamada Y, Nishida T, Ichihara S, et al: Identification of chromosome $3 \mathrm{q} 28$ and $A L P K 1$ as susceptibility loci for chronic kidney disease in Japanese individuals by a genome-wide association study. J Med Genet 50: 410-418, 2013.

28. Yamada Y, Matsuo H, Segawa T, et al: Assessment of genetic risk for myocardial infarction. Thromb Haemost 96: 220-227, 2006.

29. Fujimaki T, Kato K, Yoshida T, et al: Association of genetic variants with myocardial infarction in Japanese individuals with chronic kidney disease. Thromb Haemost 101: 963-968, 2009.

30. Oguri M, Kato K, Yokoi K, et al: Association of genetic variants with myocardial infarction in Japanese individuals with metabolic syndrome. Atherosclerosis 206: 486-493, 2009.

31. Yamada Y, Matsui K, Takeuchi I, Oguri M and Fujimaki T: Association of genetic variants with hypertension in a longitudinal population-based genetic epidemiological study. Int J Mol Med (In press).

32. Shimokata S, Oguri M, Fujimaki T, Horibe H, Kato K and Yamada Y: Association between polymorphisms of the $\alpha$-kinase 1 gene and type 2 diabetes mellitus in community-dwelling individuals. Biomed Rep 1: 940-944, 2013.

33. Ueyama C, Horibe H, Fujimaki T, Oguri M, Kato K and Yamada Y: Association of genetic variants of CELSR1 and 3q28 with hypertension in community-dwelling individuals. Biomed Rep 1: 840-844, 2013.

34. Oguri M, Fujimaki T, Horibe H, Kato K, Ichihara S and Yamada Y: Association of a polymorphism of BTN2A1 with chronic kidney disease in community-dwelling individuals. Biomed Rep 1: 868-872, 2013.

35. Fujimaki T, Horibe H, Oguri M, Kato K and Yamada Y: Association of genetic variants of the $\alpha$-kinase 1 gene with myocardial infarction in community-dwelling individuals. Biomed Rep 2: 127-131, 2014

36. Horibe H, Ueyama C, Fujimaki T, Oguri M, Kato K, Ichihara S and Yamada Y: Association of a polymorphism of BTN2A1 with dyslipidemia in community-dwelling individuals. Mol Med Rep 9: 808-812, 2014.

37. Murakata Y, Fujimaki $\mathrm{T}$ and Yamada $\mathrm{Y}$ : Association of a butyrophilin, subfamily 2 , member A1 gene polymorphism with hypertension. Biomed Rep 2: 818-822, 2014.

38. Itoh Y, Mizuki N, Shimada T, Azuma F, Itakura M, Kashiwase K, Kikkawa E, Kulski JK, Satake M and Inoko H: High-throughput DNA typing of HLA-A, -B, -C and -DRB1 loci by a PCR-SSOP-Luminex method in the Japanese population. Immunogenetics 57: 717-729, 2005.

39. Gibbons RD, Hedeker D and DuToit S: Advances in analysis of longitudinal data. Annu Rev Clin Psychol 6: 79-107, 2010.

40. Heine M, Cramm-Behrens CI, Ansari A, Chu HP, Ryazanov AG, Naim HY and Jacob R: $\alpha$-kinase 1, a new component in apical protein transport. J Biol Chem 280: 25637-25643, 2005

41. Wang SJ, Tu HP, Ko AM, Chiang SL, Chiou SJ, Lee SS, Tsai YS, Lee CP and Ko YC: Lymphocyte $\alpha$-kinase is a gout-susceptible gene involved in monosodium urate monohydrate-induced inflammatory responses. J Mol Med Berl 89: 1241-1251, 2011.

42. Newman DJ, Thakkar H, Edwards RG, Wilkie M, White T, Grubb AO and Price CP: Serum cystatin C measured by automated immunoassay: A more sensitive marker of changes in GFR than serum creatinine. Kidney Int 47: 312-318, 1995. 
43. Singh D, Whooley MA, Ix JH, Ali S and Shlipak MG: Association of cystatin $\mathrm{C}$ and estimated GFR with inflammatory biomarkers: The Heart and Soul Study. Nephrol Dial Transplant 22: 1087-1092, 2007.

44. Shlipak MG, Katz R, Cushman M, Sarnak MJ, Stehman-Breen C, Psaty BM, Siscovick D, Tracy RP, Newman A and Fried L: Cystatin-C and inflammatory markers in the ambulatory elderly. Am J Med 118: 1416, 2005

45. Wang GN, Sun K, Hu DL, Wu HH, Wang XZ and Zhang JS Serum cystatin $C$ levels are associated with coronary artery disease and its severity. Clin Biochem 47: 176-181, 2014.

46. Dandana A, Gammoudi I, Chalghoum A, Chahed H, Addad F, Ferchichi S and Miled A: Clinical utility of serum cystatin C in predicting coronary artery disease in patients without chronic kidney disease. J Clin Lab Anal 28: 191-197, 2014.

47. Qing X, Furong W, Yunxia L, Jian Z, Xuping W and Ling G Cystatin $\mathrm{C}$ and asymptomatic coronary artery disease in patients with metabolic syndrome and normal glomerular filtration rate. Cardiovasc Diabetol 11: 108, 2012.

48. Patel D, Ahmad S, Silverman A and Lindsay J: Effect of cystatin $\mathrm{C}$ levels on angiographic atherosclerosis progression and events among postmenopausal women with angiographically decompensated coronary artery disease (from the Women's Angiographic Vitamin and Estrogen [WAVE] study). Am J Cardiol 111: 1681-1687, 2013.

49. Koenig W, Twardella D, Brenner H and Rothenbacher D: Plasma concentrations of cystatin $\mathrm{C}$ in patients with coronary heart disease and risk for secondary cardiovascular events: More than simply a marker of glomerular filtration rate. Clin Chem 51: 321-327, 2005.

50. Woitas RP, Kleber ME, Meinitzer A, et al: Cystatin C is independently associated with total and cardiovascular mortality in individuals undergoing coronary angiography. The Ludwigshafen Risk and Cardiovascular Health (LURIC) study. Atherosclerosis 229: 541-548, 2013.

51. Ni L, Lü J, Hou LB, Yan JT, Fan Q, Hui R, Cianflone K, Wang W and Wang DW: Cystatin C, associated with hemorrhagic and ischemic stroke, is a strong predictor of the risk of cardiovascular events and death in Chinese. Stroke 38: 3287-3288, 2007.

52. Seliger SL, Longstreth WT Jr, Katz R, et al: Cystatin C and subclinical brain infarction. J Am Soc Nephrol 16: 3721-3727, 2005

53. Stenina OI, Byzova TV, Adams JC, McCarthy JJ, Topol EJ and Plow EF: Coronary artery disease and the thrombospondin single nucleotide polymorphisms. Int J Biochem Cell Biol 36 : 1013-1030, 2004.
54. Majack RA, Goodman LV and Dixit VM: Cell surface thrombospondin is functionally essential for vascular smooth muscle cell proliferation. J Cell Biol 106: 415-422, 1988.

55. Kyriakides TR, Zhu YH, Smith LT, et al: Mice that lack thrombospondin 2 display connective tissue abnormalities that are associated with disordered collagen fibrillogenesis, an increased vascular density, and a bleeding diathesis. J Cell Biol 140: 419-430, 1998

56. Yang Z, Kyriakides TR and Bornstein P: Matricellular proteins as modulators of cell-matrix interactions: Adhesive defect in thrombospondin 2-null fibroblasts is a consequence of increased levels of matrix metalloproteinase-2. Mol Biol Cell 11: 3353-3364, 2000.

57. Yang Z, Strickland DK and Bornstein P: Extracellular matrix metalloproteinase 2 levels are regulated by the low density lipoprotein-related scavenger receptor and thrombospondin 2. J Biol Chem 276: 8403-8408, 2001.

58. Topol EJ, McCarthy J, Gabriel S, et al: Single nucleotide polymorphisms in multiple novel thrombospondin genes may be associated with familial premature myocardial infarction. Circulation 104: 2641-2644, 2001.

59. Boekholdt SM, Trip MD, Peters RJ, Engelen M, Boer JM, Feskens EJ, Zwinderman AH, Kastelein JJ and Reitsma PH: Thrombospondin-2 polymorphism is associated with a reduced risk of premature myocardial infarction. Arterioscler Thromb Vasc Biol 22: e24-e27, 2002.

60. Ehret GB, O'Connor AA, Weder A, Cooper RS and Chakravarti A: Follow-up of a major linkage peak on chromosome 1 reveals suggestive QTLs associated with essential hypertension: GenNet study. Eur J Hum Genet 17: 1650-1657, 2009.

61. Shriner D, Adeyemo A and Rotimi CN: Joint ancestry and association testing in admixed individuals. PLoS Comput Biol 7: e1002325, 2011.

62. Smith NL, Chen MH, Dehghan A, et al; Wellcome Trust Case Control Consortium: Novel associations of multiple genetic loci with plasma levels of factor VII, factor VIII, and von Willebrand factor: The CHARGE (Cohorts for Heart and Aging Research in Genome Epidemiology) Consortium. Circulation 121: 1382-1392, 2010.

63. Tang W, Schwienbacher C, Lopez LM, et al: Genetic associations for activated partial thromboplastin time and prothrombin time, their gene expression profiles, and risk of coronary artery disease. Am J Hum Genet 91: 152-162,2012. 